\title{
Trajectory Optimization for Chance-Constrained Nonlinear Stochastic Systems
}

\author{
Yashwanth Kumar Nakka and Soon-Jo Chung
}

\begin{abstract}
This paper presents a new method of computing a sub-optimal solution of a continuous-time continuousspace chance-constrained stochastic nonlinear optimal control problem (SNOC) problem. The proposed method involves two steps. The first step is to derive a deterministic nonlinear optimal control problem (DNOC) with convex constraints that are surrogate to the SNOC by using generalized polynomial chaos (gPC) expansion and tools taken from chance-constrained programming. The second step is to solve the DNOC problem using sequential convex programming (SCP) for trajectory generation. We prove that in the unconstrained case, the optimal value of the DNOC converges to that of SNOC asymptotically and that any feasible solution of the constrained DNOC is a feasible solution of the chance-constrained SNOC because the gPC approximation of the random variables converges to the true distribution. The effectiveness of the gPC-SCP method is demonstrated by computing safe trajectories for a second-order planar robot model with multiplicative stochastic uncertainty entering at the input while avoiding collisions with a specified probability.
\end{abstract}

\section{INTRODUCTION}

Model-based design strategies for planning [1] and control [2] of robotic systems often take a deterministic approach with robustness guarantees [3] to quantify performance under worst-case uncertainties. These approaches assume a bounded value of uncertainty leading to conservative trajectories and control laws. Central to a confidence-based design solution for a robot or vehicle operating in dynamic environment is a systematic approach that accounts for uncertainties in the dynamic model, state and input constraints, and even state estimation of highly-nonlinear systems to guarantee safety and performance with high probability. Examples of systems that require safety guarantees under uncertainty include spacecraft with thrusters as actuators during proximity operations [4], and a quadrotor flying in turbulent winds [5].

Considering safety in conjunction with optimality becomes more difficult if the dynamics themselves are subject to stochastic noise. Such optimal motion planning or guidance problems with stochastic dynamics can be formulated as a continuous-time continuous-space stochastic nonlinear optimal control problem (SNOC) with chance constraints. Suboptimal-solution methods to solve model-based SNOC problem based on Pontryagin's minimum principle include differential dynamic programming [6] and iterative linear-quadratic-Gaussian [7]. Sampling-based methods like Monte Carlo motion planning [8] for trajectory optimization,

Yashwanth Kumar Nakka and Soon-Jo Chung are with the Department of Aerospace (GALCIT), California Institute of Technology. Email:\{ynakka@caltech.edu, sjchung@caltech.edu\}
Markov chain approximation method [9], and path-integral approach [10] can be used under certain assumptions on the cost function, dynamics, and uncertainty for systems affine in control.

In this paper, we present a novel approach to compute solution trajectories of a chance-constrained SNOC problem. The method involves deriving a deterministic nonlinear optimal control (DNOC) problem with convex constraints that are a surrogate to the SNOC problem by systematically accounting for nonlinear stochastic dynamics using generalized polynomial chaos expansions (gPC) [11], [12] and obtaining deterministic convex approximations of linear and quadratic chance constraints using tools taken from chance constrained programming [13], [14]. The DNOC problem is then solved using sequential convex programming (SCP) [15], [16] for real-time trajectory optimization. The gPC approach uses function approximation theory to model an unknown random process with basis functions that are chosen based on knowledge of the uncertainty affecting the process [11]. For example, a Hermite polynomial basis with the standard normal distribution is known to yield exponential convergence [11] if the uncertainties in the system are Gaussian. The gPC approximation is used to derive ordinary differential equations (ODEs) in terms of gPC coefficients. The DNOC problem and convex constraints are reformulated in terms of gPC coefficients as decision variables to apply a trust-regionbased SCP method to compute feasible trajectories.

The gPC expansion approach was used for stability analysis and control design of uncertain systems [17], [18], [19]. For trajectory optimization, recent work focuses on nonlinear systems with parametric uncertainty [20], [21] with no constraints on the state, or linear systems with linear chance-constraints that do not extend to the SNOC problem considered here and lack analysis on the deterministic approximation of the uncertain system. In [22], [23] linear chance constraints were considered for probabilistic optimal planning, strictly for linear systems. The literature on chance-constrained programming focuses on problems with deterministic decision variable and uncertain system parameters for both linear [13] and nonlinear [14] cases. The linear chance-constraint results can be readily transformed to the case with a random decision variable for an unknown measure. The quadratic chance constraint would lead to an inner semi-definite program [24] that adds complexity to the SNOC problem considered in this paper.

The main contribution of the present paper is hence to solve a class of optimal control problems that include both chance or probabilistic inequality constraints and stochastic 
nonlinear dynamics via gPC-SCP. In order to characterize the deterministic approximation obtained using a gPC method, we present analysis on convergence of a DNOC problem to the SNOC problem for an unconstrained case. Then, we prove that any feasible solution of the constrained DNOC problem is a feasible solution of chance-constrained SNOC problem with an appropriate gPC transformation step applied. This proves that solving a DNOC problem ensures constraint satisfaction with the specified probability. In order to bound the deviation of the random variable from its mean, we derive a conservative deterministic quadratic constraint approximation of the quadratic chance-constraint using multivariate Chebyshev inequality [25], which can be used to find trajectories that have a specified variance.

The paper is organized as follows. We discuss the stochastic nonlinear optimal control (SNOC) problem with results on deterministic approximations of chance constraints along with preliminaries on gPC expansions in Sec. II. The deterministic surrogate of the SNOC problem in terms of the gPC coefficients and a SCP formulation of the DNOC problem are presented with analysis in Sec. III. In Sec. IV, we apply the proposed gPC-SCP method for computing a safe trajectory for Dubin's second-order model with specified probability for avoiding collisions and discuss the limitations of the method. We conclude the paper in Sec. V with brief discussion on the results of the analysis and the application of the gPC-SCP method.

\section{Problem AND PREliminaries}

\section{A. Stochastic Nonlinear Optimal Control Problem}

In this section, we present the finite-horizon stochastic nonlinear optimal control problem with joint chance constraints in continuous time and continuous space. The problem considered here has an expectation cost function which is quadratic in the random state variable $x(t)$ and the deterministic control policy $\bar{u}(t)$. The evolution of the stochastic process $x(t)$ for all sampled paths is defined by a stochastic differential equation. The joint chance constraints guarantee constraint feasibility with a probability of $1-\epsilon$, where $\epsilon>0$ and is chosen to be a small value (example: $\epsilon \in[0.001,0.05])$ for better constraint satisfaction. The following optimal control problem is considered with the state and control as the decision variables.

$$
\begin{aligned}
J^{*}=\min _{x(t), \bar{u}(t)} & \mathrm{E}\left[\int_{t_{0}}^{t_{f}} \mathcal{L}(x(t), \bar{u}(t)) d t+\mathcal{L}_{f}\left(x\left(t_{f}\right)\right)\right] \\
\text { s.t. } & \dot{x}(t)=f(t, x(t), \bar{u}(t))+g(t, x(t), \bar{u}(t)) \theta(t) \\
& \operatorname{Pr}(x(t) \in \mathbb{F}) \geq 1-\epsilon \\
& \bar{u}(t) \in \mathbb{U} \quad \forall t \in\left[t_{0}, t_{f}\right] \\
& x\left(t_{0}\right)=x_{0} \quad \mathrm{E}\left(x\left(t_{f}\right)\right)=\hat{x}_{f} \\
& \theta(t) \in \mathcal{N}\left(\hat{\theta}(t), \Sigma_{\theta}(t)\right) t \in\left[t_{0}, t_{f}\right]
\end{aligned}
$$

where the cost functional $\mathcal{L}$ and the terminal cost $\mathcal{L}_{f}$ are defined as

$$
\begin{aligned}
\mathcal{L}(x(t), \bar{u}(t)) & =x(t)^{\top} Q x(t)+\bar{u}(t)^{\top} R \bar{u}(t) \\
\mathcal{L}_{f}\left(x\left(t_{f}\right)\right) & =x\left(t_{f}\right)^{\top} Q_{f} x\left(t_{f}\right)
\end{aligned}
$$

The matrices $Q$ and $Q_{f}$ are positive semi-definite and $R$ is a positive definite matrix. In the following, we define each of the aforementioned elements of the problem (1) and discuss convex approximations of linear and quadratic chance constraints.

1) Stochastic Differential Equation (SDE) [26]: The dynamics of the system is modeled as a controlled diffusion process with Ito assumptions. The random variable $x(t)$ is defined on a probability space $(\Omega, \mathcal{F}, \operatorname{Pr})$ where $\Omega$ is the sample space, $\mathcal{F}$ forms a $\sigma$-field with measure $\operatorname{Pr}$.

$$
\begin{aligned}
& d x(t)=f(t, x(t), \bar{u}(t)) d t+g(t, x(t), \bar{u}(t)) d w(t), \\
& \operatorname{Pr}\left(\left|x\left(t_{0}\right)-x_{0}\right|=0\right)=1, \quad \forall t_{0} \leq t \leq t_{f}<\infty
\end{aligned}
$$

where: $f(.,):. \mathbb{R}^{+} \times \mathbb{S} \times \mathbb{U} \rightarrow \mathbb{R}^{d_{x}}, g(.,):. \mathbb{R}^{+} \times \mathbb{S} \times \mathbb{U} \rightarrow$ $\mathbb{R}^{d_{x} \times d_{\theta}}$, and $w(t)$ is a $d_{\theta}$-dimensional Wiener process and the initial random variable $x_{0}$ is independent of $w(t)-w\left(t_{0}\right)$ for $t \geq t_{0}$. The sets $\mathbb{S} \subseteq \mathbb{R}^{d_{x}}$ and $\mathbb{U} \subseteq \mathbb{R}^{d_{u}}$ are compact sets.

Assumption 1. The functions $f(t, x(t), \bar{u}(t))$ and $g(t, x(t), \bar{u}(t))$ are defined and measurable on $\mathbb{R}^{+} \times \mathbb{S} \times \mathbb{U}$.

Assumption 2. Equation (3) has a unique solution $x(t)$, that is continuous with probability 1 , and $\exists$ a $K \in \mathbb{R}^{++}$such that the following conditions are satisfied:

a) Lipschitz condition: $\forall t \in\left[t_{0}, t_{f}\right],\left(x_{j}, \bar{u}_{j}\right) \in \mathbb{S} \times \mathbb{U}, j=$ 1,2 ,

$$
\begin{aligned}
& \left\|f\left(t, x_{1}, \bar{u}_{1}\right)-f\left(t, x_{2}, \bar{u}_{2}\right)\right\|+\left\|g\left(t, x_{1}, \bar{u}_{1}\right)-g\left(t, x_{2}, \bar{u}_{2}\right)\right\|_{F} \\
& \quad \leq K\left\|\left(x_{1}, \bar{u}_{1}\right)-\left(x_{2}, \bar{u}_{2}\right)\right\|
\end{aligned}
$$

b) Restriction on growth: $\forall t \in\left[t_{0}, t_{f}\right],\left(x_{1}, \bar{u}_{1}\right) \in \mathbb{S} \times \mathbb{U}$

$$
\left\|f\left(t, x_{1}, \bar{u}_{1}\right)\right\|^{2}+\left\|g\left(t, x_{1}, \bar{u}_{1}\right)\right\|_{F}^{2} \leq K^{2}\left(1+\left\|\left(x_{1}, \bar{u}_{1}\right)\right\|^{2}\right)
$$

The noise $\theta(t)=\frac{d w}{d t}$ is assumed to be driven by a Gaussian process with known mean $\hat{\theta}(t)$ and variance $\Sigma_{\theta}(t)$ that are bounded piece-wise continuous functions of time $t$.

2) Control Policy: The deterministic control policy is motivated by a hardware implementation strategy, where a state dependent Markov control policy defined on the compact set $\mathbb{S}$ is sampled for a value with highest probability (or) for the mean.

Assumption 3. The control policy $\bar{u}(t) \in \mathbb{U} \subseteq \mathbb{R}^{d_{u}}$ is deterministic and the set $\mathbb{U}$ is a convex set.

3) Chance Constraints [13]: The feasible region or set $\mathbb{F}$ is given by the linear and quadratic inequality constraint:

$$
\mathbb{F}=\left\{x(t) \in \mathbb{S}: f_{i}(x(t)) \leq 0 \forall i \in\{1, \ldots, m\}\right\},
$$

which can be relaxed by the chance constraint set with a guaranteed constraint satisfaction probability of $1-\epsilon$

$$
\Xi^{\mathrm{CC}}=\{x(t) \in \mathbb{S}: \operatorname{Pr}(x(t) \in \mathbb{F}) \geq 1-\epsilon\}
$$

A convex relaxation of the chance constraint for an arbitrary distribution of the state vector $x(t)$ due to the nonlinearity in the system is intractable, so an extension of the problem called Distributionally Robust Chance Constraints (DRCC) given as follows,

$$
\Xi^{\mathrm{DRCC}}=\left\{x(t) \in \mathrm{S}: \inf _{x(t) \sim\left(\hat{x}, \Sigma_{x}\right)} \operatorname{Pr}(x(t) \in \mathbb{F}) \geq 1-\epsilon\right\}
$$


where the chance constraint is satisfied for all distributions with known mean and variance of the decision variable is used. The set defined by the DRCC in (8) is a conservative approximation [14] of the chance constraint i.e., $\Xi^{\mathrm{DRCC}} \subseteq$ $\Xi^{\mathrm{CC}}$.

a) Distributionally Robust Linear Constraint (DRLC) [13]: Consider a single linear chance constraint with $a \in \mathbb{R}^{n}$ and $b \in \mathbb{R}:$

$$
\Xi^{\mathrm{LCC}}=\left\{x(t) \in \mathbb{S}: \operatorname{Pr}\left(a^{\top} x(t)+b \leq 0\right) \geq 1-\epsilon\right\}
$$

Assuming that the mean $\hat{x}$ and the covariance $\Sigma_{x}$ of $x$ are known, a distributionally robust constraint version of (9) is given as

$\Xi^{\mathrm{DRLC}}=\left\{x(t) \in \mathbb{S}: \inf _{x(t) \sim\left(\hat{x}, \Sigma_{x}\right)} \operatorname{Pr}\left(a^{\top} x(t)+b \leq 0\right) \geq 1-\epsilon\right\}$

Equivalently, (10) can be rewritten in the following deterministic form, which will be later used to derive a second-order cone constraint for the DNOC:

$\Xi^{\mathrm{DRLC}}=\left\{x(t) \in \mathbb{S}: a^{\top} \hat{x}(t)+b+\sqrt{\frac{1-\epsilon}{\epsilon}} \sqrt{a^{\top} \Sigma_{x} a} \leq 0\right\}$

Remark 1. The set defined in (11) is a subset of the original constraint in (9) i.e., $\Xi^{\mathrm{DRLC}} \subseteq \Xi^{\mathrm{LCC}}$, See [13] for the proof.

If the dynamics is linear, constraint in (11) is replaced with a much tighter equivalent deterministic constraint given by the following equation

$\Xi^{\mathrm{GLCC}}=\left\{x(t) \in \mathbb{S}: a^{\top} \hat{x}(t)+b+\operatorname{erf}(1-2 \epsilon) \sqrt{a^{\top} \Sigma_{x} a} \leq 0\right\}$

where the function $\operatorname{erf}($.$) is defined as \operatorname{erf}(\delta)=\frac{2}{\sqrt{\pi}} \int_{0}^{\delta} e^{-t^{2}} d t$ and the constraint set $\Xi^{\mathrm{GLCC}}$ is transformed to a secondorder cone constraint for $\epsilon \in(0,0.5)$.

b) Quadratic Constraint: Proposition 1 presents a new conservative deterministic relaxation for the quadratic chance constraint that can be used to bound the deviation of the random vector $x(t)$ from the mean $\hat{x}(t)$.

Proposition 1. The constraint set

$$
\Xi^{\mathrm{DQ}}=\left\{x(t) \in \mathbb{S}: \frac{1}{c} \operatorname{tr}\left(A \Sigma_{x}\right) \leq \epsilon\right\}
$$

is a conservative approximation of the original quadratic chance constraint

$$
\Xi^{\mathrm{CCQ}}=\left\{x(t) \in \mathbb{S}: \operatorname{Pr}\left((x-\hat{x})^{\top} A(x-\hat{x}) \geq c\right) \leq \epsilon\right\}
$$

i.e., $\Xi^{\mathrm{DQ}} \subseteq \Xi^{\mathrm{CCQ}}$, where $A \in \mathbb{R}^{n \times n}$ is a positive definite matrix and $c>0 \in \mathbb{R}$ and $\Sigma_{x}(t)$ is the covariance of the random variable $x(t)$ at time $t$.

Proof: We will prove that any random vector $x \in \mathbb{S}$ that is in the set $\Xi^{\mathrm{DQ}}$ is also in the set $\Xi^{\mathrm{CCQ}}$ implying $\Xi^{\mathrm{DQ}} \subseteq$ $\Xi^{\mathrm{CCQ}}$. The two sets are defined by the following inequalities respectively. The proof follows from the approach taken to prove the multivariate Chebyschev's inequality [25]. Let $F(x)$ be the Cumulative Distribution Function (CDF) of the random variable $x$ and $v=x-\hat{x}$.

$$
\mathbb{G}=\left\{v \in \mathbb{S}: v^{\top} A v \geq c\right\} \Longrightarrow \frac{1}{c} v^{\top} A v \geq 1 \forall v \in \mathbb{G}
$$

Using the definition of probability in terms of the CDF,

$$
\begin{aligned}
\operatorname{Pr}((x-\hat{x}) \in \mathbb{G}) & \leq \frac{1}{c} \int_{v \in \mathbb{G}} v^{\top} \operatorname{AvdF}(v) \\
& \leq \frac{1}{c} \int_{v \in \mathbb{R}^{n}} v^{\top} \operatorname{AvdF}(v)
\end{aligned}
$$

Let $a_{i j}$ be an element of matrix $A$ in the $i^{\text {th }}$ row and $j^{\text {th }}$ column, and $v_{i}$ be the $i^{\text {th }}$ element in the vector $v$. Using the expansion $v^{\top} A v=\sum_{i=1}^{n} \sum_{j=1}^{n} a_{i j} v_{i} v_{j}$ in the inequality above, the integral is simplified.

$$
\begin{aligned}
\int_{v \in \mathbb{R}^{n}} v^{\top} A v d F(v) & =\int_{v \in \mathbb{R}^{v}} \sum_{i=1}^{n} \sum_{j=1}^{n} a_{i j} v_{i} v_{j} d F(v) \\
& =\sum_{i=1}^{n} \sum_{j=1}^{n} a_{i j} \int_{v \in \mathbb{R}^{n}} v_{i} v_{j} d F(v) \\
& =\operatorname{tr}\left(A \Sigma_{x}\right)
\end{aligned}
$$

Now consider the inequality in (13). The quadratic chance constraint holds if (13) is satisfied, as $\operatorname{Pr}((x-\hat{x}) \in \mathbb{G}) \leq$ $\frac{1}{c} \operatorname{tr}\left(A \Sigma_{x}\right)$. Therefore, (13) is a conservative deterministic approximation of the quadratic chance constraint $\operatorname{Pr}((x-$ $\left.\hat{x})^{\top} A(x-\hat{x}) \geq c\right) \leq \epsilon$ i.e., $\Xi^{\mathrm{DQ}} \subseteq \Xi^{\mathrm{CCQ}}$. Note that if $\epsilon$ is a design variable, the approximation can be made tight by solving an inner semi-definite program following the approach in [24].

c) Joint Chance Constraints (JCC) [14]: The joint quadratic chance constraints are split into multiple individual constraints using Boole's inequality.

$$
\begin{gathered}
\inf _{x(t) \sim\left(\hat{x}, \Sigma_{x}\right)} \operatorname{Pr}\left(\wedge_{i=1}^{m} a_{i}^{\top} x+b_{i} \leq 0\right) \geq 1-\epsilon \\
\Longleftrightarrow \sup _{x(t) \sim\left(\hat{x}, \Sigma_{x}\right)} \operatorname{Pr}\left(\vee_{i=1}^{m} a_{i}^{\top} x+b_{i} \geq 0\right) \leq \epsilon \\
\subseteq \sum_{i=1}^{m} \sup _{x(t) \sim\left(\hat{x}, \Sigma_{x}\right)} \operatorname{Pr}\left(a_{i}^{\top} x+b_{i} \geq 0\right) \leq \epsilon \\
\inf _{x(t) \sim\left(\hat{x}, \Sigma_{x}\right)} \operatorname{Pr}\left(a_{i}^{\top} x+b_{i} \leq 0\right) \geq 1-\epsilon_{i}
\end{gathered}
$$

The distributionally robust joint chance constraints (DRJCC) are split into multiple single chance constraints using Bonferroni's inequality [14] method in (16) with naive risk allocation. Equations (16), (17) show the steps for DRJCC using Bonferroni's inequality, a similar approach is followed for JCC using Boole's inequality [22]. The risk $\epsilon$ is split between all the $m$ constraints equally $\sum_{i=1}^{m} \epsilon_{i}=\epsilon$ such that we get $m$ individual DRCC of the form given in (17). The risk allocation can be tightened by solving a two stage optimization problem as discussed in [27].

\section{B. Wiener-Askey Polynomial Chaos ([11], [12], [20])}

The generalized Polynomial Chaos (gPC) expansion theory is used to model uncertainty with finite second-order moments as a series expansion of orthogonal polynomials. The polynomials are orthogonal with respect to a known density function $\rho($.$) . Consider the random vector \xi$ with independent identically distributed (iid) random variables $\left\{\xi_{i}\right\}_{i=1}^{d_{\xi}}$ as elements. Each $\xi_{i}$ is normally distributed with 
zero mean and unit variance. The random vector $x(t)$, defined by the SDE in (3) can be expressed as the following series of polynomials

$$
x_{i}(t)=\sum_{j=0}^{\infty} x_{i j}(t) \phi_{j}(\xi)
$$

where $x_{i}$ denote the $i^{\text {th }}$ element in the vector $x \in \mathbb{S}$ and $x_{i j}$ is the $j^{\text {th }}$ coefficient in the series expansion. The dimension $d_{\xi}$ is the sum of number of random inputs in the SDE (3) and the number of random initial conditions. The functions $\phi_{j}(\xi)$ are constructed using Hermite polynomial [11] basis such that are orthogonal with respect to the joint probability density function $\rho(\xi)=\varrho\left(\xi_{1}\right) \varrho\left(\xi_{2}\right) \cdots \varrho\left(\xi_{d \xi}\right)$, where $\varrho\left(\xi_{k}\right)=\frac{1}{\sqrt{2 \pi}} e^{\frac{-\xi_{k}^{2}}{2}}$. We refer to [28] for details on construction of the polynomials. The series expansion is truncated to a finite number $\ell+1$ as $x \approx \sum_{j=0}^{\ell} x_{i j}(t) \phi_{j}(\xi)$ based on the maximum degree of the polynomials required to represent the variable $x$.

$$
x_{i j}(t)=\frac{\int_{\mathbb{D}} \rho(\xi) x_{i}(t) \phi_{j}(\xi) d \xi}{\left\langle\phi_{j}(\xi), \phi_{j}(\xi)\right\rangle}
$$

The coefficients $x_{i j}(t)$ are computed using the Galerkin projection given in (19), where $\left\langle\phi_{i}(\xi), \phi_{j}(\xi)\right\rangle=\int_{\mathbb{D}} \rho(\xi) \phi_{i}(\xi) \phi_{j}(\xi) d \xi$.

$$
\int_{\mathbb{D}} \rho(\xi) x_{i}(t) \phi_{j}(\xi) d \xi=\sum_{k=1}^{m} w_{k} \rho\left(n_{k}\right) x_{i}(t) \phi_{j}\left(n_{k}\right)
$$

For non-polynomial functions the Galerkin projection can be approximately computed using Stochastic collocation [12] method as shown in (20) using Gauss-Hermite quadrature, where $n_{k}$ are quadrature nodes and $w_{k}$ are the respective node weights. In the following section, we derive an approximate nonlinear ordinary differential equation system for the SDE in (3) using gPC expansion and the Galerkin scheme.

Lemma 1. (Cameron-Martin Theorem [29]) The gPC series approximation in (18) converges to the true value $x_{i} \in \mathcal{L}_{2}$.

$\left\|x_{i}(t)-\sum_{j=0}^{\ell} x_{i j}(t) \phi_{j}(\xi)\right\|_{\mathcal{L}_{2}} \rightarrow 0$, as $\ell \rightarrow \infty \forall t \in\left[t_{0}, t_{f}\right]$

Remark 2. The expectation $\mathrm{E}\left(x_{i}\right)$ and variance $\Sigma_{x_{i}}$ of the random variable $x_{i}$ can be expressed in terms of the coefficients of the expansion as given below

$$
\mathrm{E}\left(x_{i}\right)=x_{i 0} \quad \Sigma_{x_{i}} \approx \sum_{j=1}^{\ell} x_{i j}^{2}\left\langle\phi_{j}, \phi_{j}\right\rangle \text { as } \ell \rightarrow \infty
$$

Lemma 1 and Remark 2 will be used in studying the convergence of the gPC approximation of the cost function, the SDE and the chance constraints. Furthermore, the higherorder moments can be expressed as a polynomial function of the coefficients.

\section{DETERMinistic SURROGATE OF THE SNOC PROBLEM}

The stochastic nonlinear optimal control problem discussed in Sec. II-A is reformulated in terms of the coefficients of the gPC expansion, with decision variables as the gPC coefficients and the control $\bar{u}$. In the following, we discuss the existence and uniqueness of a solution to the coupled Ordinary Differential Equations (ODE) obtained form gPC approximation of SDE, and present the convex constraints for the gPC coefficients obtained from deterministic approximation of chance constraints. The main theorem that discusses the convergence and feasibility of the approximation is presented at the end of this section.

\section{A. Deterministic ODE Approximation of the SDE}

The gPC expansion in (18) is applied for all the elements in the vector $x \in \mathbb{S} \subseteq \mathbb{R}^{d_{x}}$ and the matrix representation using Kronecker product is given in the following, where

$$
\begin{aligned}
& X=\left[\begin{array}{lllllll}
x_{10} & \cdots & x_{1 \ell} & \cdots & x_{d_{x} 0} & \cdots & x_{d_{x} \ell}
\end{array}\right]^{\top} \\
& \Phi(\xi)=\left[\begin{array}{lll}
\phi_{0}(\xi) & \cdots & \phi_{\ell}(\xi)
\end{array}\right]^{\top} \\
& x \approx \bar{\Phi} X ; \text { where } \bar{\Phi}=\mathbb{I}_{d_{x} \times d_{x}} \otimes \Phi(\xi)^{\top}
\end{aligned}
$$

The dynamics of the coefficients $x_{i j}$ with the above notation is given in (25), where: $f_{i}$ and $g_{i}$ are the $i^{\text {th }}$ element of the vector $f(t, x, \bar{u})$ and $i^{\text {th }}$ row of the matrix $g(t, x, \bar{u})$ respectively.

$$
\begin{aligned}
\dot{x}_{i j}(t) & =\bar{f}_{i j}(t, X, \bar{u})+\bar{g}_{i j}\left(t, X, \bar{u}, \hat{\theta}, \Sigma_{\theta}\right) \\
\bar{f}_{i j} & =\frac{\int_{\mathbb{D}} \rho(\xi) \phi_{j}(\xi) f_{i}(t, \bar{\Phi} X, \bar{u}) d \xi}{\left\langle\phi_{j}(\xi), \phi_{j}(\xi)\right\rangle} \\
\bar{g}_{i j} & =\frac{\int_{\mathbb{D}} \rho(\xi) \phi_{j}(\xi) g_{i}(t, \bar{\Phi} X, \bar{u}) \theta(\xi, t) d \xi}{\left\langle\phi_{j}(\xi), \phi_{j}(\xi)\right\rangle} \\
\theta(\xi, t) & =\hat{\theta}(t) \phi_{0}(\xi)+\sqrt{\Sigma_{\theta}}(t) \phi_{1}(\xi)
\end{aligned}
$$

The full nonlinear ODE with the stacked vector $X$ is given as

$$
\dot{X}=\bar{f}(t, X, \bar{u})+\bar{g}\left(t, X, \bar{u}, \hat{\theta}(t), \Sigma_{\theta}(t)\right)
$$

The sequential convex programming method used for trajectory optimization involves linearization of the dynamics about a given trajectory and discretization for time integration. The existence and uniqueness of solution to the ODE surrogate ensure convergence of any Picard iteration scheme used for integration. In Proposition 2, we will present conditions for existence and uniqueness of solution to the ODE in (25).

Proposition 2. The ODE system in (25) obtained using $g P C$ approximation of the SDE has a solution and the solution is unique assuming that the SDE satisfies the existence and uniqueness conditions in (4), (5) and the expectation in (27) is bounded for each $j=0,1, \cdots, \ell$, where: $P=$ $\left[\begin{array}{ll}\bar{\Phi} & 0 \\ 0 & \mathbb{I}\end{array}\right], k_{j}=\left\langle\phi_{j}, \phi_{j}\right\rangle, L_{f_{j}}(\xi)=\left|\phi_{j}(\xi)\right|\|P\|_{2}, L_{g_{j}}(\xi)=$ $L_{f_{j}}(\xi)\left|\phi_{1}(\xi)\right|$ and $K_{f_{i j}}=\frac{K}{k_{j}} \mathrm{E}\left(L_{f_{j}}(\xi)\right)$.

$$
K_{g_{i j}}=\|\hat{\theta}(t)\|_{2} K_{f_{i j}}+\frac{K}{k_{j}}\left\|\Sigma_{\theta}(t)\right\|_{2} \mathrm{E}\left(L_{g_{j}}(\xi)\right)
$$

Proof: The existence and uniqueness is proved by showing local Lipschitz continuity of the functions $f_{i j}, g_{i j}$ in (25) w.r.t $(X, \bar{u}) \quad \forall t \in\left[t_{0}, t_{f}\right]$ in the ball $\mathbb{B}=\left\{(X, \bar{u}) \in \mathbb{R}^{d_{x}+d_{u}} \mid\left\|(X, \bar{u})-\left(X_{0}, \bar{u}_{0}\right)\right\|_{2} \leq r\right\}$ 
where $\left(X_{0}, \bar{u}_{0}\right)$ is the initial condition for each $i j$ and piecewise continuity w.r.t $t$. The piece-wise continuity w.r.t $t$ is implied due to growth condition in (5) that bounds $\bar{f}_{i}$ and $\bar{g}_{i}$ uniformly with respect to $t \in\left[t_{0}, t_{f}\right]$, see [26]. Let $y_{1}=\left(X_{1}, \bar{u}_{1}\right)$ and $y_{2}=\left(X_{2}, \bar{u}_{2}\right) \in \mathbb{S} \times \mathbb{U}$ and $g_{i 1}-g_{i 2}=g_{i}\left(t, \bar{\Phi} X_{1}, \bar{u}_{1}\right)-g_{i}\left(t, \bar{\Phi} X_{2}, \bar{u}_{2}\right)$.

$$
\begin{aligned}
&\left|\bar{g}_{i j}\left(t, y_{1}\right)-\bar{g}_{i j}\left(t, y_{2}\right)\right| \leq \frac{1}{k_{j}} \int_{\mathbb{D}}\left|\rho(\xi) \phi_{j}(\xi)\left(g_{i 1}-g_{i 2}\right) \theta\right| d \xi \\
&\left|\bar{g}_{i j}\left(t, y_{1}\right)-\bar{g}_{i j}\left(t, y_{2}\right)\right| \leq K_{g_{i j}}\left\|y_{1}-y_{2}\right\|_{2} \\
&\left|\bar{f}_{i j}\left(t, y_{1}\right)-\bar{f}_{i j}\left(t, y_{2}\right)\right| \leq K_{f_{i j}}\left\|y_{1}-y_{2}\right\|_{2}
\end{aligned}
$$

In the inequality (28), using the boundedness of $f$ and $g$ in (4), the Cauchy-Schwartz inequality, the sub-multiplicative property of norms, and $\|\cdot\|_{2} \leq\|\cdot\|_{F}$, we obtain the inequality (29). The inequality (30) can be proved following same steps as shown above. Therefore, if the integrals in (27) are bounded the functions $\bar{g}_{i j}$ and $\bar{f}_{i j}$ are locally Lipschitz continuous.

\section{B. Convex Approximation of the Chance Constraint}

The deterministic approximation of the chance constraints discussed in the Sec. II-A are expressed in terms of the gPC coefficients that define a feasible set for the deterministic optimal control problem with gPC coefficients as decision variables.

Lemma 2. The second-order cone constraint given below

$$
\left(a^{\top} \otimes M\right) X+b+\sqrt{\frac{1-\epsilon}{\epsilon}} \sqrt{X^{\top} U N N^{\top} U^{\top} X} \leq 0
$$

is equivalent to the deterministic approximation of the DRLC in (10) as $\ell \rightarrow \infty$., where the matrices $M, U, N$ are given by

$$
\begin{aligned}
M & =\left[\begin{array}{llll}
1 & 0 & \cdots & 0
\end{array}\right]_{1 \times(\ell+1)} \\
U & =\left[\begin{array}{ccc}
a_{1} & 0 & 0 \\
0 & \ddots & 0 \\
0 & 0 & a_{d_{x}}
\end{array}\right] \otimes \mathbb{I}_{(\ell+1) \times(\ell+1)} \\
N & =\mathbb{1}_{d_{x} \times d_{x}} \otimes \mathbb{H} ; \quad \mathbb{H}=\left[\begin{array}{cc}
0 & \mathbb{O} \\
\mathbb{O} & \sqrt{\mathrm{E}\left(H H^{\top}\right)}
\end{array}\right]
\end{aligned}
$$$$
\text { where } H=\left[\begin{array}{lll}
\phi_{1}(\xi) & \cdots & \phi_{\ell}(\xi)
\end{array}\right]^{\top}
$$

and $\mathbb{1}$ is a matrix with entries as 1 .

Proof: It is sufficient to prove that $\left(a^{\top} \otimes M\right) \approx a^{\top} \hat{x}$ and $X^{\top} U N N^{\top} U^{\top} X \approx a^{\top} \Sigma_{x} a$ as $\ell \rightarrow \infty$. Invoking Lemma 1 and Remark 2, the polynomials of gPC coefficients can be replaced by mean and variable of the variable $x$.

$$
\begin{aligned}
& \left(a^{\top} \otimes M\right) X=\left[\begin{array}{llll}
a_{1} M & a_{2} M & \cdots & a_{d_{x}} M
\end{array}\right] X \\
& =a_{1} x_{10}+a_{2} x_{20}+\cdots+a_{d_{x}} x_{d x 0} \\
& \approx a^{\top} \hat{x}
\end{aligned}
$$

Equation (33) shows the steps involved to prove $\left(a^{\top} \otimes M\right) \approx$ $a^{\top} \hat{x}$. Let us define a vector $p_{i}=\left[\begin{array}{ll}x_{i 0} & \bar{p}_{i}^{\top}\end{array}\right]^{\top}$ where $\bar{p}_{i}=$ $\left[\begin{array}{lll}x_{i 1} & \cdots & x_{i \ell}\end{array}\right]^{\top}$.

$$
U^{\top} X=\left[\begin{array}{llll}
a_{1} p_{1}^{\top} & a_{2} p_{2}^{\top} & \cdots & a_{d_{x}} p_{d_{x}}^{\top}
\end{array}\right]^{\top}
$$

$$
\begin{aligned}
& N N^{\top} U^{\top} X=\left[\begin{array}{llll}
\mathbb{H} a_{1} p_{1} & \mathbb{H} a_{2} p_{2} & \cdots & \mathbb{H} a_{d_{x}} p_{d_{x}}
\end{array}\right] \\
& \begin{aligned}
X^{\top} U N N^{\top} U^{\top} X & =\sum_{i=1}^{d_{x}} \sum_{j=1}^{d_{x}} a_{i} a_{j} p_{i}^{\top} \mathbb{H} p_{j} \\
& =\sum_{i=1}^{d_{x}} \sum_{j=1}^{d_{x}} a_{i} a_{j} \bar{p}_{i}^{\top} \mathrm{E}\left(H H^{\top}\right) \bar{p}_{j} \\
& \approx a^{\top} \Sigma_{x} a
\end{aligned}
\end{aligned}
$$

Using this notation, the matrices in (31) are expanded as shown in (34), (35), and (36). Therefore the equivalence is proved by Lemma 1 as $\ell \rightarrow \infty$.

Lemma 3. The quadratic inequality

$$
\sum_{i=1}^{d_{x}} \sum_{k=1}^{\ell} a_{i}\left\langle\phi_{k}, \phi_{k}\right\rangle x_{i k}^{2} \leq \epsilon c
$$

expressed in terms of the gPC coefficients is equivalent to the $S D P$ constraint in (13) as $\ell \rightarrow \infty$ given that $A$ is a diagonal matrix with $i^{\text {th }}$ diagonal element as $a_{i}$.

Proof: Consider the deterministic approximation $\operatorname{tr}\left(A \Sigma_{x}\right) \leq c \epsilon$ of the quadratic constraint, we can expand it as follows.

$$
\begin{aligned}
\operatorname{tr}\left(A \Sigma_{x}\right) \leq c \epsilon & \equiv \sum_{i=1}^{d_{x}} a_{i} \mathrm{E}\left(\left(x_{i}-\hat{x}_{i}\right)\left(x_{i}-\hat{x}_{i}\right)\right) \leq c \epsilon \\
& \equiv \sum_{i=1}^{d_{x}} \sum_{j=1}^{\ell} a_{i}\left\langle\phi_{j}, \phi_{j}\right\rangle x_{i j}^{2} \leq c \epsilon
\end{aligned}
$$

The equivalence is proved by directly expanding the trace and using Remark 2 as shown in (38).

\section{Cost Function}

Remark 3. The expectation cost function defined in (1) with the functions $\mathcal{L}$ defined in (2) expressed in terms of the gPC coefficients is given as the following, using the notation in (24).

$$
\begin{aligned}
\mathcal{L}_{d}(X(t), \bar{u}(t)) & =X(t)^{\top} \bar{\Phi}^{\top} Q \bar{\Phi} X(t)+\bar{u}(t)^{\top} R \bar{u}(t) \\
\mathcal{L}_{d f}\left(X\left(t_{f}\right)\right) & =X\left(t_{f}\right)^{\top} \bar{\Phi}^{\top} Q_{f} \bar{\Phi} X\left(t_{f}\right)
\end{aligned}
$$

The procedure can be extended to any polynomial cost function in terms of state.

Theorem 1. The deterministic nonlinear optimal control problem (DNOC) with convex constraints given below

$$
\begin{array}{cl}
J_{d}^{*}=\min _{X(t), \bar{u}(t)} & \int_{t_{0}}^{t_{f}} \mathcal{L}_{d}(X(t), \bar{u}(t)) d t+\mathcal{L}_{d f}\left(X\left(t_{f}\right)\right) \\
\text { s.t. } & \text { Equations }\{(26),(31),(37)\} \\
& \bar{u}(t) \in \mathbb{U} \quad \forall t \in\left[t_{0}, t_{f}\right] \\
& X\left(t_{0}\right)=X_{0} \quad X\left(t_{f}\right)=X_{f}
\end{array}
$$

is an approximate surrogate for the stochastic nonlinear optimal control (SNOC) problem in (1) with following being true:

(a) In the case with no chance constraints, the cost $\mid J_{d}^{*}-$ 
$J^{*} \mid \rightarrow 0$ as $\ell \rightarrow \infty$

(b) In the case with linear and quadratic chance constraints, any feasible solution of problem DNOC is a feasible solution of SNOC as $\ell \rightarrow \infty$, assuming that a feasible solution exists.

Proof: Case (a): It is sufficient to prove that the cost function and the dynamics are exact as $\ell \rightarrow \infty$. Using the Kronecker product notation, due to Lemma 1, we have the following

$$
\begin{gathered}
\|x-\bar{\Phi} X\|_{\mathcal{L}_{2}} \rightarrow 0 \text { as } \ell \rightarrow \infty \\
(41) \Longrightarrow\|\dot{x} \rightarrow \bar{\Phi} \dot{X}\|_{\mathcal{L}_{2}} \rightarrow 0 \text { as } \ell \rightarrow \infty \\
(41) \Longrightarrow \quad\left|\mathcal{L}_{d}-\mathcal{L}\right| \rightarrow 0 \text { as } \ell \rightarrow \infty \\
\quad\left|\mathcal{L}_{d f}-\mathcal{L}_{f}\right| \rightarrow 0 \text { as } \ell \rightarrow \infty
\end{gathered}
$$

From (42), (43), we can conclude that since the cost function, the dynamics and the initial and terminal conditions are exact as $\ell \rightarrow \infty$, the optimal value $\left|J_{d}^{*}-J^{*}\right| \rightarrow 0$ as $\ell \rightarrow \infty$.

Case (b): Consider the sets $\Xi^{\mathrm{LgPC}}$, and $\Xi^{\mathrm{QgPC}}$ defined below.

$$
\begin{aligned}
& \Xi^{\mathrm{LgPc}}=\{x \in \mathbb{S}: x \approx \bar{\Phi} X \text { where } X \in(31)\} \\
& \Xi^{\mathrm{QgPC}}=\{x \in \mathbb{S}: x \approx \bar{\Phi} X \text { where } X \in(37)\}
\end{aligned}
$$

Using Lemmas 2 and 3, we have the approximate convex constraints converge to the deterministic equivalent of the distributionally robust chance constraint as $\ell \rightarrow \infty$.

$$
\begin{aligned}
& \text { Lemma } 2 \Longrightarrow \Xi^{\mathrm{LgPc}}-\Xi^{\mathrm{DRLC}} \sim \emptyset \text { as } \ell \rightarrow \infty \\
& \text { Lemma } 3 \Longrightarrow \Xi^{\mathrm{QgPC}}-\Xi^{\mathrm{DC}} \sim \emptyset \text { as } \ell \rightarrow \infty
\end{aligned}
$$

Using Remark 1 and Proposition 1 we have the following

$$
\begin{gathered}
\text { Remark } 1 \Longrightarrow \Xi^{\mathrm{DRLC}} \subseteq \Xi^{\mathrm{LCC}} \\
\text { Proposition } 1 \Longrightarrow \Xi^{\mathrm{DC}} \subseteq \Xi^{\mathrm{CCQ}}
\end{gathered}
$$

Combining (46) and (47), we can conclude that (48) holds as $\ell \rightarrow \infty$. This proves that if a feasible solution exists for the DNOC in (40) then it is a feasible solution of the SNOC in (1) as $\ell \rightarrow \infty$.

\section{Sequential Convex Programming}

The approximate deterministic optimal control problem is solved using sequential convex programming (SCP) for trajectory optimization. In the SCP method, we reformulate the NDOP as a convex optimization problem by linearizing the nonlinear dynamics about a given trajectory that forms a set of linear constraints on the state and control action. The linear constraints and the integral cost function are discretized for $\mathrm{N}$ time steps between the time horizon $\left[t_{0}, t_{f}\right]$ such that the state and action variables at each time step act as decision variables of the convex optimization as follows:

$$
\dot{X}^{(i+1)}=A^{(i)} X^{(i+1)}+B^{(i)} \bar{u}^{(i+1)}+Z^{(i)}
$$

$$
\begin{aligned}
& A^{(i)}=\left.\frac{\partial(\bar{f}+\bar{g})}{\partial X}\right|_{\left(X^{(i-1)}, \bar{u}^{(i-1)}\right)} ; \quad B^{(i)}=\left.\frac{\partial(\bar{f}+\bar{g})}{\partial \bar{u}}\right|_{\left(X^{(i-1)}, \bar{u}^{(i-1)}\right)} \\
& Z^{(i)}=\bar{f}\left(X^{(i-1)}, \bar{u}^{(i-1)}\right)+\bar{g}\left(X^{(i-1)}, \bar{u}^{(i-1)}\right) \\
& -A^{(i)} X^{(i-1)}-B^{(i)} \bar{u}^{(i-1)} \\
& X^{(i)}[k+1]=A^{(i)}[k] X^{(i)}[k]+B^{(i)}[k] \bar{u}^{(i)}[k]+Z^{(i)}[k] \\
& A^{(i)}[k]=e^{A^{(i)} \Delta t} \quad B^{(i)}[k]=\int_{0}^{\Delta t} e^{A^{(i)} \tau} B^{(i)} d \tau \\
& Z^{(i)}[k]=\int_{0}^{\Delta t} e^{A^{(i)} \tau} Z^{(i)} d \tau
\end{aligned}
$$

The discretized dynamics (51), reformulated chance constraints (31), (37), the initial and terminal conditions are used as constraints at each iteration.

$$
\begin{array}{ll}
\min _{X^{(i)}, \bar{u}^{(i)}} & \left.\sum_{k=1}^{N} \mathcal{L}_{d}\left(X^{(i)}[k]\right), \bar{u}^{(i)}[k]\right) \Delta t+\mathcal{L}_{d f}\left(X^{(i)}[N]\right) \\
\text { s.t. } \quad & \text { Equations }\{(51),(31),(37)\} \\
& \bar{u}^{(i)}[k] \in \mathbb{U} \quad \forall k \in[1, N] \\
& X^{(i)}[1]=X_{0} \quad X^{(i)}[N]=X_{f} \\
& \left\|X^{i}-X^{(i-1)}\right\|_{2} \leq \alpha \beta^{i} \forall k
\end{array}
$$

Equation (53) shows the SCP formulation of the DNOC problem given trajectory of $(i-1)^{\text {th }}$ iteration with the constraint set at each time step $k$ and iteration $i$. An additional constraint $\left\|X^{(i)}-X^{(i-1)}\right\| \leq \alpha \beta^{i}$ called trust region is used to handle infeasibility of the sub-convex problem, where $\alpha>0$ and $\beta \in(0,1)$. The trust region shrinks as the number of iterations increases which acts as a convergence criterion. The SCP algorithm is known to converge to the KKT point of the DNOC problem under mild conditions. For detailed analysis on convergence, see [15], [16].

\section{Example: Motion Planning With Obstacles}

In this section, we discuss the implementation of the gPCSCP approach on the following unicycle dynamic model:

$$
\left[\begin{array}{c}
\ddot{x} \\
\ddot{y} \\
\ddot{\theta}
\end{array}\right]=\left[\begin{array}{cc}
\cos \theta & 0 \\
\sin \theta & 0 \\
0 & 1
\end{array}\right]\left[\begin{array}{l}
(1+\lambda) \bar{u}_{1} \\
(1+\lambda) \bar{u}_{2}
\end{array}\right]
$$

where $(x, y)$ is the position of the centre of mass with respect to an inertial frame and $\theta$ is the heading angle.

$$
0 \leq \bar{u}_{1} \leq 1 \quad\left|\bar{u}_{2}\right| \leq 10
$$

The dynamics is underactuated with a nonlinear control input matrix and has a Gaussian multiplicative uncertainty term entering at the input $\lambda \sim \mathcal{N}\left(0, \Sigma_{\lambda}\right)$ with zero mean and known variance.

$$
x\left(t_{0}\right)=[0 ; 0.4 ; 0 ; 0 ; 0] ; \quad \mathrm{E}\left(x\left(t_{f}\right)\right)=[10 ; 0.4 ; 0 ; 0 ; 0]
$$

The initial and terminal conditions are given in (56). In the scenario considered here, the robotic car needs to compute a sub-optimal dynamically feasible, safe trajectory from initial state to the expected terminal state in (56), while avoiding collision with the obstacle and the wall with specified probability. We have the knowledge of the initial 
state with probability 1 and only know the expected state information at the terminal state. The environment map and initial conditions were carefully chosen such that a feasible trajectory exists between the wall and the obstacle for low variance simulations. For the situation with high variance of $\lambda$ the gPC-SCP method computes a feasible solution that lies on the other side of the obstacle.

gPC Form of the Dynamics: The nonlinear functions cos and $\sin$ are approximated by the following technique, often used for Galerkin projection of harmonic functions.

$$
\begin{aligned}
\cos \theta & \approx \cos \left(\theta_{0}+\sum_{j=1}^{\ell} \theta_{j} \phi_{j}\right) \\
& \approx \cos \theta_{0} \cos \left(\sum_{j=1}^{\ell} \theta_{j} \phi_{j}\right)-\sin \theta_{0} \sin \left(\sum_{j=1}^{\ell} \theta_{j} \phi_{j}\right)
\end{aligned}
$$

In the above equation, we use Taylor series approximation for the sin and cos of the higher order terms $\sum_{j=1}^{\ell} \theta_{j} \phi_{j}$ for computing the Galerkin projection. We use the first four Hermite polynomials for the gPC expansion. The computation of the $\mathrm{gPC}$ form and the Galerkin projection was done using Matlab. For the sake of brevity we leave out the gPC form of the dynamics from this paper. The Gaussian uncertainty in the system can be expressed in terms of the Hermite polynomials, where $\xi \in \mathcal{N}(0,1)$.

$$
\lambda(\xi)=\hat{\lambda} \phi_{0}(\xi)+\sqrt{\Sigma}_{\lambda} \phi_{1}(\xi)
$$

The controls in this particular example are assumed to be deterministic $\bar{u}_{i}=\bar{u}_{i} \phi_{0}(\xi)$ as $\phi_{0}=1$.

Chance Constraints: The joint chance constraint for collision checking with the wall and the obstacle is give in the following

$$
\operatorname{Pr}\left(x_{2}(t)<2 ;\left\|p(t)-p_{\text {obs }}\right\|_{2} \geq R_{\text {safe }}\right) \geq 1-\epsilon
$$

The position of the robot and obstacle are $p(t)=$ $\left[x_{1}(t), x_{2}(t)\right]$, the position $p_{\text {obs }}=[5,0]$ respectively. The radius of the obstacle is given by $R_{\mathrm{safe}}=1.2$. The joint chance constraints are split into individual chance constraints using Bonferroni's inequality with $\epsilon_{1}+\epsilon_{2}=\epsilon$ given value of $\epsilon$. We use naive risk allocation such that $\epsilon_{1}=\epsilon_{2}=0.005$ given that $\epsilon=0.01$.

$$
\operatorname{Pr}\left(x_{2}(t)>2\right) \leq \epsilon_{1} ; \operatorname{Pr}\left(\left\|p(t)-p_{\text {obs }}\right\|_{2} \leq R_{\text {safe }}\right) \leq \epsilon_{2}
$$

The individual chance constraints are given in (60). In the SCP approach, for each iteration $i$ the second constraint for collision avoidance, $\left\|p(t)-p_{\text {obs }}\right\|_{2} \geq R_{\text {safe }}$ is linearized about position trajectory $\bar{p}^{(i-1)}(t)$ using the technique in [15], [16], where $i$ is the iteration of the SCP.

$$
\begin{gathered}
\operatorname{Pr}\left(x_{2}(t) \geq 2\right) \leq \epsilon_{1} \quad \operatorname{Pr}\left(a^{\top} p(t) \geq b\right) \leq \epsilon_{2} \\
\quad x_{2}^{i}+\sqrt{\frac{1-\epsilon_{1}}{\epsilon_{1}}} \sqrt{\Sigma_{x_{2}}^{i}} \leq 2 \\
a^{\top} \hat{p}^{(i)}(t)+\sqrt{\frac{1-\epsilon_{2}}{\epsilon_{2}}} \sqrt{a^{\top} \Sigma_{p(t)}^{i} a} \leq b
\end{gathered}
$$

Equation (61) shows the linear chance constraints for collision checking with this approach and (62) shows the distributionally robust convex approximation in terms of the mean and variance of the variables $x_{2}(t)$ and $p(t)$ at each time $t$. The second-order constraints are expressed in terms of the $\mathrm{gPC}$ coefficients for the gPC-SCP method, where $a=p_{\text {obs }}-\bar{p}^{(i-1)}(t)$ and $b=\left(x_{\mathrm{obs}}-\bar{p}^{(i-1)}(t)\right)^{\top} p_{\mathrm{obs}}-R_{\mathrm{safe}}\left\|\bar{p}^{(i-1)}(t)-x_{\mathrm{obs}}\right\|_{2}$.

SCP Implementation and Simulation Details: For the SCP implementation, we choose a time horizon of $10 \mathrm{sec}-$ onds for robot to move from initial point to the terminal point with $N_{\text {steps }}=50$ time steps. The continuous ODE dynamics obtained from the gPC transformation was linearized and then discretized and imposed as constraints for all the time steps. The constraints in (62) and the control at each time step $t$ are already convex, that are directly used in the SCP algorithm.

Results: The trajectory optimization was run with an initial trajectory as the line joining initial to the terminal point for two situations $\Sigma_{\lambda}=\left\{0.01^{2}, 0.05^{2}\right\}$. We run the algorithm for following cost functions 1) control effort i.e., $R=I$ and $Q_{f}=Q=0$ as the cost function for $\Sigma_{\lambda}=\left\{0.01^{2}, 0.05^{2}\right\}$ shown in Fig. 1 as trajectories T1 and T2 respectively, 2) both control and state penalized in the cost function i.e., $R=I$ and $Q=I$ for $\Sigma_{\lambda}=0.05^{2}$ shown as trajectory T3 in Fig. 1 .

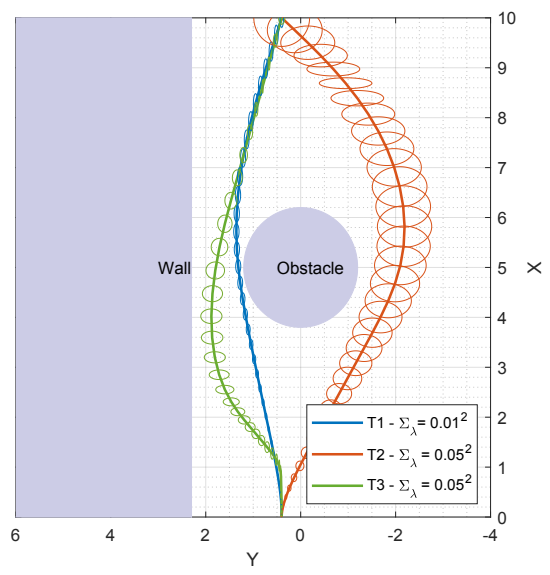

Fig. 1: Plot showing mean of the state and uncertainty ellipse corresponding to $3 \sigma$ confidence for $\epsilon=0.05$.

Note that when we minimize both state and control cost we can find a safe trajectory T3 between the wall and the obstacle. The trajectories T1 and T2 were compared in Fig. 2 with the open-loop simulations of the original dynamics in (54) using the policy computed by gPC-SCP approach on the approximated dynamics. Using the openloop deterministic policy the robot reaches the terminal state with out collision.

Limitations: The Galerkin scheme used in the paper is computationally expensive, to use the gPC-SCP technique for higher-dimensional problems, numerical methods like stochastic collocation need to be implemented for Galerkin projection. The problem formulation would be same with the stochastic collocation method. The SCP problem requires a good initialization for faster convergence. For longer time 


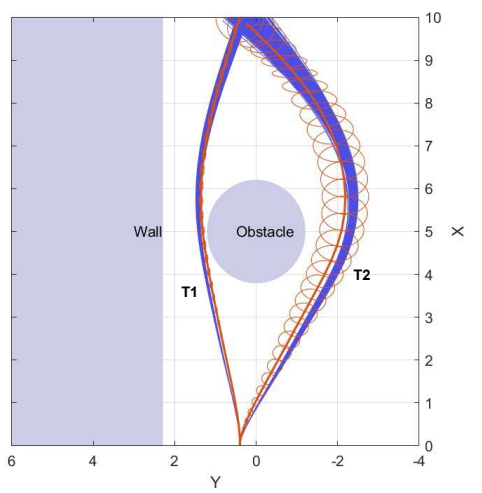

Fig. 2: Plot showing comparison of $\mathrm{T} 1$ and $\mathrm{T} 2$ trajectories with open-loop policy simulations.

horizon problems multi-element polynomial chaos approach has to be used for uncertainty propagation to achieve better accuracy.

\section{CONCLusion}

The paper presented an approximate deterministic surrogate for the stochastic nonlinear optimal control problem with chance constraints for stochastic trajectory optimization. The approach used generalized polynomial chaos (gPC) to derive a deterministic ordinary differential equation for the stochastic differential equation and a deterministic cost function for the expectation cost function. The main theorem showed that a feasible solution of the deterministic optimal control problem is a feasible solution of the stochastic nonlinear optimal control problem. The proposed gPC-SCP is applied to an example problem to obtain a suboptimal feasible trajectory that is guaranteed to avoid collision with the specified probability. The effectiveness of the method is validated by comparing the trajectories obtained from the method with open-loop runs for multiple realizations of stochastic uncertainty using the open-loop control policy obtained.

\section{ACKNOWLEDGEMENT}

This work was in part funded by the Jet Propulsion Laboratory, California Institute of Technology and the Raytheon Company.

\section{REFERENCES}

[1] LaValle, S. M., Planning Algorithms, Cambridge University Press, 2006.

[2] Spong, M. W., Hutchinson, S., and Vidyasagar, M., Robot modeling and control, 2006

[3] Zhou, K., Doyle, J. C., and Glover, K., Robust and optimal control, Vol. 40, Prentice hall New Jersey, 1996.

[4] Nakka, Y. K., Foust, R. C., Lupu, E. S., Elliott, D. B., Crowell, I. S., Chung, S.-J., and Hadaegh, F. Y., "Six degree-of-freedom spacecraft dynamics simulator for formation control research," AAS/AIAA Astrodynamics Specialist Conference, 2018.

[5] Shi, G., Shi, X., O'Connell, M., Yu, R., Azizzadenesheli, K., Anandkumar, A., Yue, Y., and Chung, S.-J., "Neural lander: Stable drone landing control using learned dynamics," IEEE International Conference on Robotics and Automation (ICRA), 2019.
[6] Tassa, Y., Mansard, N., and Todorov, E., "Control-limited differential dynamic programming," IEEE International Conference on Robotics and Automation (ICRA), 2014, pp. 1168-1175.

[7] Todorov, E. and Li, W., "A generalized iterative LQG method for locally-optimal feedback control of constrained nonlinear stochastic systems," Proceedings of the 2005, American Control Conference, 2005., 2005, pp. 300-306.

[8] Janson, L., Schmerling, E., and Pavone, M., "Monte Carlo motion planning for robot trajectory optimization under uncertainty," Robotics Research, Springer, 2018, pp. 343-361.

[9] Kushner, H. J., "Numerical methods for stochastic control problems in continuous time," SIAM Journal on Control and Optimization, Vol. 28, No. 5, 1990, pp. 999-1048.

[10] Kappen, H. J., "Linear theory for control of nonlinear stochastic systems," Physical review letters, Vol. 95, No. 20, 2005, pp. 200201.

[11] Xiu, D. and Karniadakis, G. E., "The Wiener-Askey polynomial chaos for stochastic differential equations," SIAM journal on scientific computing, Vol. 24, No. 2, 2002, pp. 619-644.

[12] Xiu, D., "Fast numerical methods for stochastic computations: a review," Communications in computational physics, Vol. 5, No. 2-4, 2009, pp. 242-272.

[13] Calafiore, G. C. and El Ghaoui, L., "On distributionally robust chanceconstrained linear programs," Journal of Optimization Theory and Applications, Vol. 130, No. 1, 2006, pp. 1-22.

[14] Zymler, S., Kuhn, D., and Rustem, B., "Distributionally robust joint chance constraints with second-order moment information," Mathematical Programming, Vol. 137, No. 1-2, 2013, pp. 167-198.

[15] Morgan, D., Chung, S.-J., and Hadaegh, F. Y., "Model predictive control of swarms of spacecraft using sequential convex programming," Journal of Guidance, Control, and Dynamics, Vol. 37, No. 6, 2014, pp. $1725-1740$.

[16] Morgan, D., Chung, S.-J., and Hadaegh, F., "Spacecraft swarm guidance using a sequence of decentralized convex optimizations," AIAA/AAS Astrodynamics Specialist Conference, 2012, p. 4583.

[17] Hover, F. S. and Triantafyllou, M. S., "Application of polynomial chaos in stability and control," Automatica, Vol. 42, No. 5, 2006, pp. 789795.

[18] Fisher, J. and Bhattacharya, R., "Stability Analysis of Stochastic Systems using Polynomial Chaos," Proc. American Control Conference, 2008, pp. 4250-4255.

[19] Kim, K.-K., Shen, D. E., Nagy, Z. K., and Braatz, R. D., "Wiener's polynomial chaos for the analysis and control of nonlinear dynamical systems with probabilistic uncertainties [historical perspectives]," IEEE Control Systems Magazine, Vol. 33, No. 5, 2013, pp. 58-67.

[20] Boutselis, G. I., Pan, Y., De La Tore, G., and Theodorou, E. A., "Stochastic trajectory optimization for mechanical systems with parametric uncertainties," arXiv preprint arXiv:1705.05506, 2017.

[21] Fisher, J. and Bhattacharya, R., "Optimal Trajectory Generation with Probabilistic System Uncertainty using Polynomial Chaos," Journal of Dynamic Systems, Measurement, and Control, Vol. 133, No. 1, 2011, pp. 014501.

[22] Blackmore, L., Ono, M., Bektassov, A., and Williams, B. C., "A probabilistic particle-control approximation of chance-constrained stochastic predictive control," IEEE transactions on Robotics, Vol. 26, No. 3, 2010, pp. 502-517.

[23] Blackmore, L., Ono, M., and Williams, B. C., "Chance-constrained optimal path planning with obstacles," IEEE Transactions on Robotics, Vol. 27, No. 6, 2011, pp. 1080-1094.

[24] Vandenberghe, L., Boyd, S., and Comanor, K., "Generalized Chebyshev bounds via semidefinite programming," SIAM review, Vol. 49, No. 1, 2007, pp. 52-64.

[25] Chen, X., "A new generalization of Chebyshev inequality for random vectors," arXiv preprint arXiv:0707.0805, 2007.

[26] Arnold, L., Stochastic differential equations, 1974.

[27] Ono, M. and Williams, B. C., "Iterative Risk Allocation: A new approach to robust Model Predictive Control with a joint chance constraint," 2008 47th IEEE Conference on Decision and Control, Dec 2008, pp. 3427-3432.

[28] Ghanem, R. G. and Spanos, P. D., Stochastic finite elements: a spectral approach, Courier Corporation, 2003.

[29] Cameron, R. H. and Martin, W. T., "The orthogonal development of non-linear functionals in series of Fourier-Hermite functionals," Annals of Mathematics, 1947, pp. 385-392. 